\title{
Primer reporte de Brachyplatys subaeneus (Westwood) (Heteroptera: Plataspidae) en Ecuador y el listado sinóptico de sus plantas hospedantes
}

\author{
First report by Brachyplatys subaeneus (Westwood) (Heteroptera: Plataspidae) \\ for Ecuador and the synoptic list of its host plants \\ Yostin J. Añino ${ }^{1,2}$, Martha B. Sumba-Zhongor ${ }^{3}$, Jaime A. Naranjo-Morán ${ }^{4}$, \\ Randhy Rodríguez $z^{1}$, Alonso Santos-Murgas ${ }^{1}$, Bruno Zachrisson ${ }^{5 *}$
}

RESUMEN

Se reporta por primera vez en Ecuador la presencia del chinche invasor Brachyplatys subaeneus (Westwood, 1837) proveniente de Asia. Esta plaga ataca leguminosas de importancia agrícola como el guandú (Cajanus cajan) y se ha detectado en otros países de América como Panamá y República Dominicana. Adicionalmente se muestra un listado sinóptico de las plantas hospederas que utiliza esta plaga como alimento.

Palabras clave: Cajanus cajan, especie invasora, plaga.

\section{ABSTRACT}

The presence of the invasive insect Brachyplatys subaeneus (Westwood, 1837) from Asia is reported for the first time in Ecuador. This pest attacks legumes of agricultural importance such as the pigeon pea (Cajanus cajan) and has been reported in other countries of America such as Panama and the Dominican Republic. Additionally, a synoptic record of host plants used by this pest as a food source is shown.

Keywords: Cajanus cajan, invasive species, pest.

\section{Introducción}

El género Brachyplatys Boisduval, 1835 proveniente de Asia fue reportado en América por Aiello et al. (2016), con individuos colectados en Panamá e identificados mediante secuencias de ADN como Brachyplatys vahlii (Fabricius, 1787). No obstante, la identificación de esta especie invasora no fue la correcta y posteriormente se definió como Brachyplatys subaeneus (Westwood, 1837), utilizando caracteres morfológicos considerados por Rédei (2016).

La investigación realizada por Añino et al. (2017) amplió el ámbito de distribución en Panamá, reportándola en la costa del Pacífico del país. PérezGelabert et al. (2019) registraron por primera vez esta especie en el Caribe y República Dominicana. Se destaca la relevancia de $B$. subaeneus, la cual se considera la segunda especie invasora en el continente americano después de Megacopta cribraria (Fabricius, 1798). Esta especie es nativa de Japón y en 1999 se confirmó su presencia en el sureste de los Estados Unidos, donde se alimentaba de plantas de la familia Fabaceae (Suiter et al., 2010). Debido a su amplia distribución y adaptación a especies de plantas hospedantes pertenecientes a diferentes familias en el continente americano, este estudio tuvo como objetivo constatar la presencia

\footnotetext{
Universidad de Panamá, Museo de Invertebrados G.B. Fairchild, Panamá, Panamá.

Universidad del Istmo, Programa de Maestría en Gestión Ambiental y Sostenibilidad, Panamá, Panamá.

Escuela Superior Politécnica del Litoral (ESPOL), Guayaquil, Ecuador.

Centro de Investigaciones Biotecnológicas del Ecuador (CIBE), Guayaquil, Ecuador.

Instituto de Investigación Agropecuaria de Panamá (IDIAP), Panamá, Panamá.

* Autor de Correspondencia: bazsalam@gmail.com
}

Fecha de Recepción: 6 de Octubre, 2019.

Fecha de Aceptación: 27 de Diciembre, 2019. 
de B. subaeneus en Ecuador. Además se consideró registrar las especies y familias de plantas que le sirven de alimento.

\section{Materiales y métodos}

Se realizaron recolecciones de Brachyplatys subaeneus en áreas cultivadas de guandú (Cajanus cajan) ubicadas en las proximidades de la Escuela Superior Politécnica del Litoral (ESPOL) Prosperina (Guayaquil, Ecuador) (29'3.526”'S; 7957'13.339'O) a $103 \mathrm{msnm}$, durante la época lluviosa (Figura 1). El nivel de infestación de los ejemplares pertenecientes a Plataspidae alcanzó un total de diez (10) individuos, recolectados el 1 de marzo de 2019. Las áreas experimentales fueron establecidas con la finalidad de implementar actividades de investigación, por lo que no se harán aplicaciones de insecticidas, para incrementar la población de la especie invasora (Figura 2). Los especímenes recolectados fueron identificados de acuerdo a la clave taxonómica de Rédei (2016) y comparados con los ejemplares de la colección de insectos del Museo de Invertebrados Graham Bell Fairchild de la Universidad de Panamá. El material biológico previamente recolectado e identificado fue almacenado en la colección entomológica de la Facultad de Ciencias de la Vida de la Escuela Superior Politécnica del Litoral (ESPOL). Posteriormente, se procedió a realizar muestreos en diferentes especies de plantas hospedantes, entre estas la Mucuna pruriens (Fabaceae) y Zea mays (Poaceae), encontradas en los predios de la Universidad Agraria del Ecuador, Guayaquil, Ecuador.

\section{Resultados y discusión}

Brachyplatys subaeneus (Westwood, 1837).

\section{Material examinado}

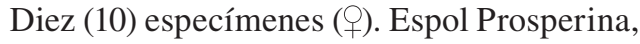
2'9'3.526”'S; 7957'13.339'O. Recolectados en marzo 1, 2019, por M. Sumba y J. Naranjo, ESPOL (Guayaquil, Ecuador).

\section{Diagnosis}

Especie relativamente pequeña de Brachyplatys (longitud del cuerpo: 4.5-5.8 $\mathrm{mm}$ ), que presenta entre sus características distintivas el disco del pronotum y la base de escutelum carente de pares de parches pequeños, redondeados y pálidos, fascias amarillas en los márgenes laterales de los esternitos abdominales (Rédei, 2016).

\section{Distribución}

Actualmente está distribuida en la región Indomalaya (Bangladesh, Camboya, China, Filipinas, India, Indonesia, Japón, Malasia, Myanmar, Singapur, Sri Lanka, Tailandia, Taiwán, Vietnam), Panamá (Aiello et al., Añino et al., 2016; Rédei, 2016) y República Dominicana (PérezGelabert et al., 2019). Además, como producto de esta investigación se reporta por primera vez en Ecuador.

\section{Hospederos}

Brachyplatys subaeneus es el único representante del género Brachyplatys y el segundo de la familia Plataspididae, después de Megacopta cribraria, presente en el continente americano. Cuenta con un registro de nueve plantas hospedantes pertenecientes a las familias Arecaceae, Araliaceae, Fabaceae y Poaceae, las cuales se encuentran distribuidas en el continente americano (Tabla 1), específicamente en las zonas tropicales.

Al menos 11 especies de Brachyplatys fueron reportadas en Asia, las cuales se alimentan de 56 especies de plantas pertenecientes a 30 géneros y 9 familias (Rider, 2015) (Tabla 1). A partir de las actualizaciones taxonómicas en plantas, se incorporaron especies de plantas hospedantes de $B$. subaeneus que incluyen a las familias Arecaceae y Araliaceae, en la región del Neotrópico (Aiello et al., 2016). De esta manera se confirma que $B$. subaeneus presenta un amplio espectro de plantas hospedantes, que incluyen 25 especies distribuidas en nueve familias (Rider, 2015). No obstante, la familia Fabaceae agrupa 15 especies de plantas hospedantes, donde este insecto invasor puede completar su ciclo biológico. Además, se destaca que $B$. subaeneus es la única especie del género que reporta plantas hospedantes de las familias Cannabaceae (Cannabis sativa L. (marihuana)), Convolvulaceae (Ipomoea batatas (L.) (batata)) y Euphorbiaceae (Acalypha bipartita, Chamaesyce parva) (Rider, 2015). Este autor corrobora que A. bipartita y C. parva (Euphorbiaceae) son hospedantes donde al menos cuatro especies del 
género Brachyplatys se consideran el primer y único reporte de esta asociación "insecto invasorplanta hospedante" (Rider, 2015).

El estudio indica que $B$. subaeneus fue colectado en Ecuador, en hojas y tallos de guandú (Cajanus cajan) (Figura 1). También se observó su presencia en otras plantas hospedantes, como la macuna (Mucuna pruriens, Fabaceae), soja (Glycine max, Leguminoseae) y maíz (Zea mays, Poaceae). Aspectos relevantes de diferentes especies de plantas hospedantes de B. subaeneus fueron considerados en esta publicación.

\section{Guandú, Cajanus cajan (Fabaceae):}

No existe reporte oficial de esta especie como hospedante de $B$. subaeneus, limitándose la asociación "insecto invasor-planta hospedante" al continente americano. Los registros obtenidos por Hoffmann (1932) se basan en fuentes desconocidas, considerando la posibilidad de que la información corresponda a otras especies de Brachyplastys (Rédei, 2016). El guandú como planta hospedante de B. subaeneus fue reportado por Aiello et al. (2016), en Panamá. Posteriormente, muestreos realizados en Panamá por Añino et al. (2017) registraron índices poblacionales elevados de $B$. subaeneus en $C$. cajan, lo que podría atribuirse a la preferencia alimenticia y/o nutricional de esta especie invasora, cuya distribución geográfica se limita al continente americano. Pérez-Gelabert et al. (2019) también confirmaron la asociación de $B$. subaeneus con el guandú como planta hospedante en República Dominicana, coincidiendo con los resultados presentados por esta investigación.

\section{Soja, Glycine $\max ($ L.) (Leguminoseae):}

El registro de B. subaeneus en soja fue reportado en el continente americano por Rédei (2016), que también detectó esta asociación biótica en Asia, específicamente en Indochina y China.

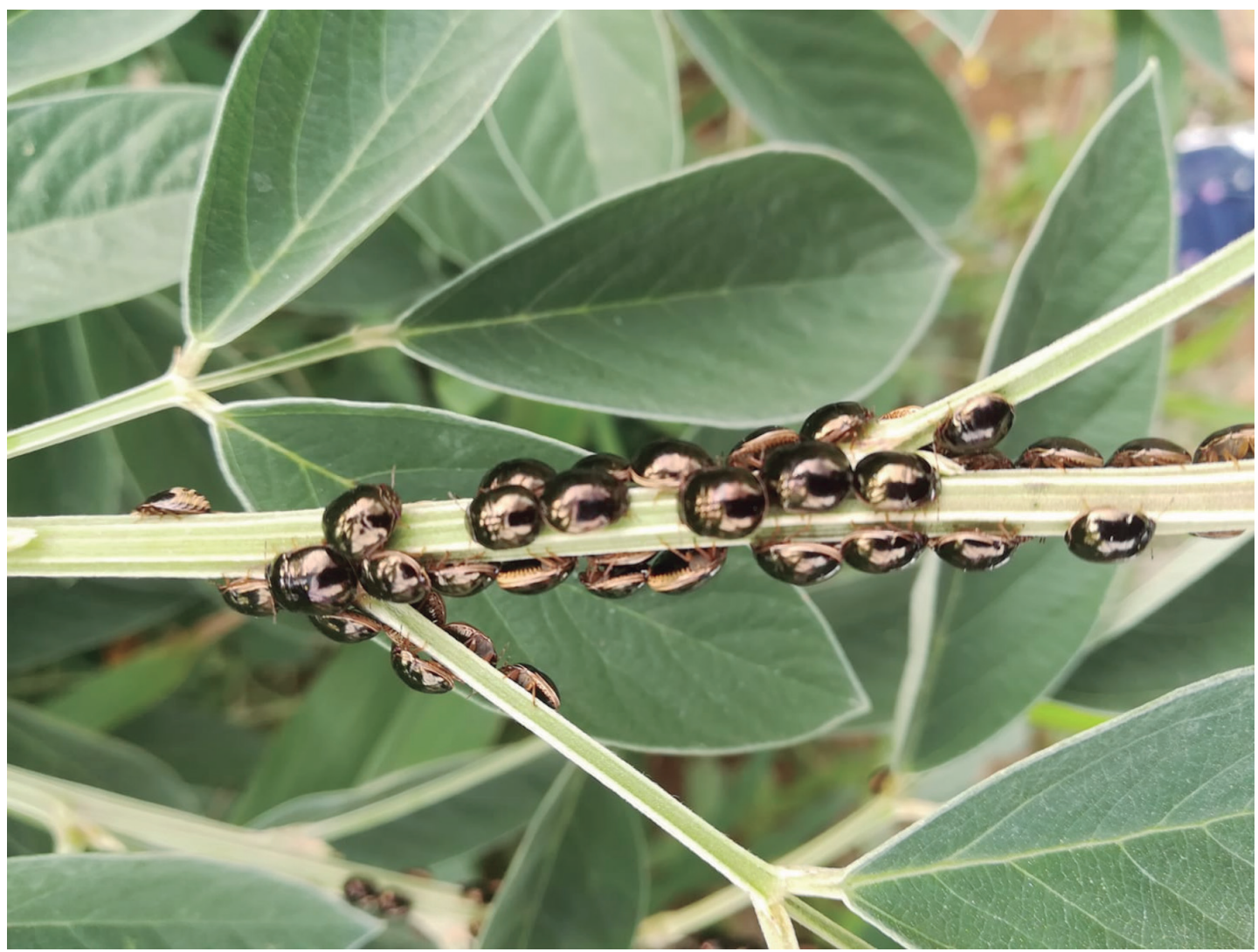

Figura 1. Adultos de Brachyplatys subaeneus (Heteroptera: Plataspidae) sobre planta de guandú (Cajanus cajan), en Prosperina, Guayaquil, Ecuador. 


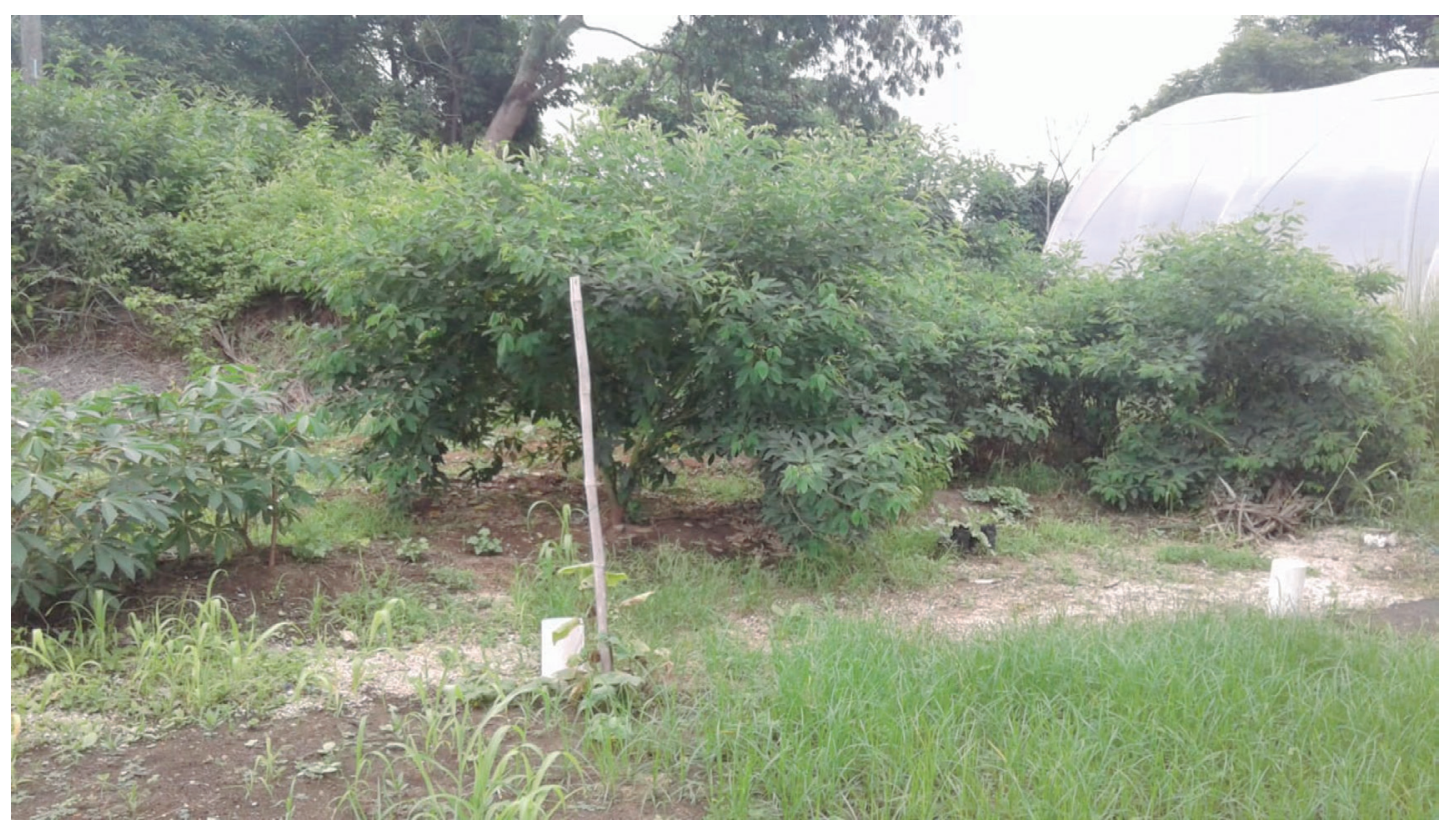

Figura 2. Áreas cultivadas de guandú (Cajanus cajan), donde se recolectó Brachyplatys subaeneus, en Prosperina, Guayaquil, Ecuador.

\section{Macuna, Mucuna pruriens (Fabaceae):}

Mucuna pruriens (Fabaceae) registró por primera vez la presencia de $B$. subaeneus en las áreas experimentales ubicadas en Ecuador. El reporte de $M$. pruriens como planta hospedante de otras especies de Brachyplatys, sin identificar, sugiere la posible adaptación de este género de Brachyplastys a ese hospedante. No obstante, otros individuos pertenecientes al género Mucuna también son considerados como especies hospedantes de Brachyplatys (Rider, 2015). Este autor menciona las asociaciones entre Muсиna poggei y Brachyplastys testudonigra, además de Mucuna aterrima como planta hospedante de Brachyplastys pacificus (Rider, 2015).

\section{Maíz, Zea mays (Poaceae):}

Se reporta por primera vez el maíz (Zea mays) como hospedante de B. subaeneus en el continente americano, considerándose la única especie de Poaceae que sirve de alimento para esta especie de insecto invasor en la región. Previamente, se había observado B. testudonigra en el cultivo de maíz (Rider, 2015). Otras especies Poaceas, como Oryza sativa L. (arroz) y Saccharum sp., probablemente S. officinarum (caña de azúcar), fueron registradas como hospedantes de B. subaeneus en Camboya, Asia (Pol et al., 2010).
Se reporta por primera vez $B$. subaeneus en Espol Prosperina (29'3.526"'S; 7957'13.339”O), para Ecuador y Sudamérica. El desconocimiento en la identificación taxonómica de esta especie invasora, aunado al reducido número de muestreos en las principales plantas hospedantes en áreas de producción de los diversos cultivos de importancia económica, podrá influir en los reportes de $B$. subaeneus en los países de América del Sur. La relación biótica de esta especie de Plataspidae con plantas hospedantes pertenecientes a diferentes familias sugiere la plasticidad de $B$. subaeneus para adaptarse a diversos ecosistemas agrícolas, condición que podría favorecer la dispersión de este insecto invasor en Sudamérica.

\section{Agradecimientos}

Agradecemos a la Facultad de Ciencias de la Vida de la Escuela Superior Politécnica del Litoral (ESPOL), Ecuador y al Museo de Invertebrados Graham Bell Fairchild de la Universidad de Panamá, por la colaboración en el presente estudio. Bruno Zachrisson agradece al Sistema Nacional de Investigación (SNI) de SENACYT, por el apoyo para el desarrollo e implementación de investigaciones que aporten al desarrollo de la entomología en Panamá. 
Tabla 1. Listado sinóptico de las plantas hospedantes de Brachyplatys subaeneus.

\begin{tabular}{|c|c|c|}
\hline Especie & Países en los que se ha reportado & Fuente \\
\hline \multicolumn{3}{|l|}{ Arecaceae } \\
\hline Bactris gasipaes & Panamá & Aiello et al., 2016 \\
\hline \multicolumn{3}{|l|}{ Araliaceae } \\
\hline Schefflera actinophylla & Panamá & Aiello et al., 2016 \\
\hline \multicolumn{3}{|l|}{ Asteraceae } \\
\hline Mikania micrantha Kunth & Macao & Rédei, 2016 \\
\hline \multicolumn{3}{|l|}{ Cannabaceae } \\
\hline Cannabis sativa $\mathrm{L}$. & India & Rédei, 2016 \\
\hline \multicolumn{3}{|l|}{ Convolvulaceae } \\
\hline Ipomoea batatas (L.) & Indochina y Archipiélago Malayo & Rédei, 2016 \\
\hline \multicolumn{3}{|l|}{ Fabaceae } \\
\hline Vachellia farnesiana (L.) & India & Rédei, 2016 \\
\hline Cajanus cajan & Ecuador, Panamá, República Dominicana & Aiello et al., 2016,Presente trabajo \\
\hline Canavalia ensiformis (L.) & Península Malaya & Rédei, 2016 \\
\hline Dolichos lablab L. & India & Rédei, 2016 \\
\hline Gliricidia sepium & Panamá, Península Malaya & Añino et al., 2017 \\
\hline Glycine $\max (\mathrm{L})$. & China, Indochina y Ecuador & Rédei, 2016 \\
\hline Indigofera suffruticosa & Desconocido & Rédei, 2016 \\
\hline Leptolobium panamense & Panamá & Aiello et al., 2016 \\
\hline Mucuna pruriens & Ecuador & Publicación actual \\
\hline Phaseolus lunatus & China e Indochina & Rédei, 2016 \\
\hline Phaseolus vulgaris L. & China, Indochina y República Dominicana & Rédei, 2016, Pérez-Gelabert et al., 2019 \\
\hline Pueraria lobata (Willd.) & Macao & Rédei, 2016 \\
\hline Pueraria phaseoloides Benth. & Sumatra (Indonesia) & Rédei, 2016 \\
\hline Vigna radiata $(\mathrm{L})$. & China e Indochina & Rédei, 2016 \\
\hline Vigna unguiculata & China, Indochina y Península Malaya & Rédei, 2016 \\
\hline \multicolumn{3}{|l|}{ Poaceae } \\
\hline Oryza sativa $\mathrm{L}$. & Cambodia & Pol et al., 2010 \\
\hline Saccharum officinarum $\mathrm{L}$. & Cambodia & Pol et al., 2010 \\
\hline Zea mays & Ecuador & Presente trabajo \\
\hline \multicolumn{3}{|l|}{ Solanaceae } \\
\hline Solanum tuberosum L. & Archipiélago Malayo & Rédei, 2016 \\
\hline \multicolumn{3}{|l|}{ Malvaceae } \\
\hline Corchorus capsularis & India & Rider, 2014 \\
\hline
\end{tabular}




\section{Literatura Citada}

Aiello, A.; Saltonstall, K.; Young, V.

2016. Brachyplatys vahlii (Fabricius, 1787), an introduced bug from Asia: first report in the Western Hemisphere (Hemiptera: Plataspidae: Brachyplatidinae). BioInvasions Records, 5 (1): 7-12.

Añino, Y.; Santos, A.; Henríquez, G.; Carranza, R.; Villareal, C. 2018. Distribución espacial del chinche invasor Brachyplatys subaeneus (Westwood, 1837) (Hemiptera: Heteroptera: Plataspidae) en Panamá. Insecta Mundi, 0630: 1-6.

Hoffmann, E.

1932. Notes on the bionomics of some oriental Pentatomidae (Hemiptera). Archivio Zoologico Italiano, 16 (3-4): 1010-1027.

Pérez-Gelabert, D.; Abud-Antún, A.; Nolasco, C.

2019. First Record of the Introduced Asian Plataspid Brachyplatys subaeneus (Westwood) (Hemiptera: Heteroptera: Plataspidae) in the Dominican Republic. Proceedings of the Entomological Society of Washington, 121(1): 135-138.
Pol, C.; Belfield, S.; Martin, R.

2010. Insects of upland crops in Cambodia. ACIAR Monograph $N^{\circ}$ 143. Australian Centre for International Agricultural Research. Canberra, Australia. 132 p.

Rédei, D.

2016. The identity of the Brachyplatys species recently introduced to Panama, with a review of bionomics (Hemiptera: Heteroptera: Plataspidae). Zootaxa, 4136 (1): 141-154.

Rider, D.

2015. Plant Host Records Plataspididae, List by plataspid Species. Disponible en: https://www.ndsu.edu/faculty/ rider/Pentatomoidea/Hosts/plant_Plataspididae.htm Consultado: 9/jul/2019.

Suiter, D.; Eger, J.; Gardner, W.; Kemerait, R.; All, J.; Roberts, P.; Greene, J.; Ames, L.; Buntin, G.; Jenkins, T.; Douce, G. 2010. Discovery and distribution of Megacopta cribraria (Hemiptera: Heteroptera: Plataspidae) in northeast Georgia. Journal of Integrated Pest Management, 1 (1): 1-4. 